\title{
On Geodesic Segments in the Infinitesimal Asymptotic Teichmüller Spaces
}

\author{
Yan $W u^{1,2}{ }^{1,}$ Qi, $^{1}$ and Zunwei Fu ${ }^{2}$ \\ ${ }^{1}$ LMIB and School of Mathematics and Systems Science, Beihang University, Beijing 100191, China \\ ${ }^{2}$ Department of Mathematics, Linyi University, Linyi 276005, China
}

Correspondence should be addressed to Zunwei Fu; zwfu@mail.bnu.edu.cn

Received 27 October 2015; Accepted 3 December 2015

Academic Editor: Stanislav Hencl

Copyright (c) 2015 Yan Wu et al. This is an open access article distributed under the Creative Commons Attribution License, which permits unrestricted use, distribution, and reproduction in any medium, provided the original work is properly cited.

Let $A Z(R)$ be the infinitesimal asymptotic Teichmüller space of a Riemann surface $R$ of infinite type. It is known that $A Z(R)$ is the quotient Banach space of the infinitesimal Teichmüller space $Z(R)$, where $Z(R)$ is the dual space of integrable quadratic differentials. The purpose of this paper is to study the nonuniqueness of geodesic segment joining two points in $A Z(R)$. We prove that there exist infinitely many geodesic segments between the basepoint and every nonsubstantial point in the universal infinitesimal asymptotic Teichmüller space $A Z(\mathbb{D})$ by constructing a special degenerating sequence.

\section{Introduction}

Let $R$ be a hyperbolic Riemann surface, that is, a Riemann surface with universal covering surface which is conformally equivalent to the open unit disk $\mathbb{D}$. Denote by $L^{\infty}(R)$ the Banach space of Beltrami differentials $\mu=\mu(z) \overline{d z} / d z$ on $X$ with finite $L_{\infty}$-norms.

Let $Q(R)$ be the space of integrable holomorphic quadratic differentials $\phi=\phi(z) d z^{2}$ on $R$ with $L_{1}$-norms:

$$
\|\phi\|=\int_{R}|\phi(z)| d x d y<+\infty
$$

Denote by $Q_{1}(R)$ the unit sphere of $Q(R)$ and by $Q_{d}(R)$ the set of all degenerating sequences of $Q(R)$. A sequence of quadratic differentials $\left\{\phi_{n}\right\}$ in $Q(R)$ is said to be a degenerating sequence if $\left\|\phi_{n}\right\|=1$ and $\phi_{n} \rightarrow 0$ locally uniformly on $R$ as $n \rightarrow \infty$.

Two elements $\mu, v \in L^{\infty}(R)$ are called infinitesimally Teichmüller equivalent, if

$$
\int_{R} \mu \phi=\int_{R} \nu \phi \quad \forall \phi \in Q(R)
$$

We denote by $[\mu]_{z}$ the infinitesimal Teichmüller equivalence class of $\mu$. The infinitesimal Teichmüller space

$$
Z(R):=\left\{[\mu]_{z}: \mu \in L^{\infty}(R)\right\}
$$

is the set of all infinitesimal Teichmüller equivalence classes of $\mu$ 's in $L^{\infty}(R)$. The point $[0]_{z}$ is called the basepoint of $Z(R)$.

A Beltrami differential $\mu \in L^{\infty}(R)$ is said to be vanishing at infinity, if, for each $\epsilon>0$, there is a compact set $E$ in $R$ such that $\left\|\left.\mu\right|_{R-E}\right\|_{\infty}<\epsilon$. Denote by $L_{0}^{\infty}(R)$ the set of all such vanishing $\mu$ 's and by

$$
Z_{0}(R):=\left\{[\mu]_{z} \in Z(R): \mu \in L_{0}^{\infty}\right\}
$$

the set of all infinitesimal Teichmüller equivalence classes of $\mu^{\prime}$ 's in $L_{0}^{\infty}(R)$.

Two elements $\mu, \nu \in L^{\infty}(R)$ are called infinitesimally asymptotically equivalent, if there exists $\sigma \in L_{0}^{\infty}(R)$ such that

$$
\int_{R} \mu \phi=\int_{R} \nu \phi+\int_{R} \sigma \phi \quad \forall \phi \in Q(R) .
$$

We denote by $[[\mu]]_{z}$ the infinitesimal asymptotic equivalence class of $\mu$. The infinitesimal asymptotic Teichmüller space

$$
A Z(R):=\left\{[[\mu]]_{z}: \mu \in L^{\infty}(R)\right\}
$$

is the set of all infinitesimal asymptotic equivalence classes of $\mu^{\prime}$ s in $L^{\infty}(R)$. The point $[[0]]_{z}$ is called the basepoint of $A Z(R)$.

It is known that $Z(R)$ and $A Z(R)$ are the tangent spaces to the classical Teichmüller space and asymptotic Teichmüller 
space at their basepoint, respectively (see [1]). For further results and properties about Teichmüller theory, we refer to the papers [1-5] and the books [6-8].

The notion of geodesics plays an important role in the study of the geometry of Teichmüller theory. A geodesic in a metric space is a continuous curve such that for any subarc its length is equal to the distance between its two endpoints. The existence and uniqueness of geodesic between two points in various spaces have been discussed for a long time (see [6, 915]). Given two points in infinitesimal Teichmüller space $Z(R)$, it is shown in [6] that there exists at least one geodesic joining them. Furthermore, it is proved in [14] that there exists precisely one geodesic segment joining the basepoint $[0]_{z}$ and $[\mu]_{z}$ in $Z(R)$ if and only if $[\mu]_{z}$ contains a uniquely extremal Beltrami coefficient with constant modulus.

The existence of geodesic joining two given points in $A Z(R)$ has been proved in $[1,6]$; however, the uniqueness of geodesic is unknown. In this paper, we will prove the nonuniqueness of geodesics in the universal infinitesimal asymptotic Teichmüller space $A Z(\mathbb{D})$ by constructing a special degenerating sequence $\left\{\phi_{n}\right\}$ in $Q(\mathbb{D})$.

The structure of this paper is as follows. Section 2 is devoted to setting up the notations and some results we need. In Section 3, a special sequence $\left\{\phi_{n}\right\}$ degenerating towards a boundary point of the unit circle is constructed. In Section 4 , it is proved that there are infinitely many geodesics joining $[[\mu]]_{z}$ with the basepoint in $A Z(\mathbb{D})$ when $[[\mu]]_{z}$ is of a nonsubstantial point using the important lemma (Lemma 2) and the constructed sequence in the previous section.

\section{Preliminaries}

In this section, we recall some notions and basic results from the Teichmüller theory. For more details, we refer to the book [6] and the paper [1].

By the Hahn-Banach and Riesz representation theorem, every element $V$ in the dual space $Q^{*}(R)$ of the Banach space $Q(R)$ of all integrable holomorphic quadratic differentials can be represented as

$$
V(\phi)=\int_{R} \mu \phi, \quad \phi \in Q(R),
$$

where $\mu$ is a Beltrami differential in $L^{\infty}(R)$. So there is natural one-to-one correspondence between the infinitesimal Teichmüller space $Z(R)$ and the dual space $Q^{*}(R)$. Thus, in what follows, the infinitesimal Teichmüller equivalence classes of Beltrami differentials on $R$ and complex linear functionals on $Q(R)$ are used for points of $Z(R)$ alternately.

For every $V \in Z(R)$, the infinitesimal extremal dilatation and the infinitesimal boundary dilatation of $V$ are defined as

$$
\begin{gathered}
\|V\|=\left\|[\mu]_{z}\right\|=\inf \left\{\|\mu\|_{\infty}: \mu \text { represents } V\right\}, \\
b(V)=b\left([\mu]_{z}\right)=\inf \left\{b^{*}(\mu): \mu \text { represents } V\right\},
\end{gathered}
$$

respectively, where

$$
\begin{aligned}
& b^{*}(\mu) \\
& \quad=\inf \left\{\left\|\left.\mu\right|_{R-E}\right\|_{\infty}: E \text { is a compact subset of } R\right\} .
\end{aligned}
$$

$\mu$ is called infinitesimal extremal if $\|\mu\|_{\infty}=\|V\|$. It is shown in [6] that $Z(R)$ is a Banach space with the infinitesimal Teichmüller norm

$$
\|V\|=\sup _{\phi \in \mathrm{Q}_{1}(R)}\left|\int_{R} \mu \phi\right|=\inf \left\{b^{*}(\mu): \mu \text { represents } V\right\} \text {. }
$$

The infinitesimal Teichmüller distance between two points $V_{1}$ and $V_{2}$ in $Z(R)$ is defined in [14]

$$
d\left(V_{1}, V_{2}\right)=\left\|V_{1}-V_{2}\right\|=\sup _{\phi \in \mathrm{Q}_{1}(R)}\left|\int_{R}\left(\mu_{1}-\mu_{2}\right) \phi\right|,
$$

where $\mu_{1}$ and $\mu_{2}$ represent $V_{1}$ and $V_{2}$, respectively.

Let $P_{z}: Z(R) \rightarrow A Z(R) ; V \mapsto \widehat{V}$ be the quotient mapping from the tangent space $Z(R)$ to the tangent space $A Z(R) . V_{1}$ and $V_{2}$ in $Z(R)$ represent the same point $\widehat{V}$ in $A Z(R)$ if $V_{1}-V_{2} \in Z_{0}(R)$. Thus, the infinitesimally asymptotic equivalence classes $[[\mu]]_{z}$ of Beltrami differentials are in oneto-one correspondence with the elements $\widehat{V}$ of $A Z(R)$ and $\mu$ represents $\widehat{V}$.

For any $\widehat{V} \in A Z(R)$, we define the quotient norm on the quotient space $A Z(R)$ as

$$
b(\widehat{V})=b\left([[\mu]]_{z}\right)=\inf \left\{b^{*}(\mu): \mu \text { represents } \widehat{V}\right\} .
$$

It is known in [6] that $b(\widehat{V})=b(V) . \mu$ is called infinitesimally asymptotically extremal if $b^{*}(\mu)=b(\widehat{V})$. Furthermore, $A Z(R)$ is a Banach space with the standard seminorm (see [6])

$$
\begin{aligned}
b(\widehat{V}) & =\sup _{\left\{\phi_{n}\right\} \in Q_{d}(R)} \limsup _{n \rightarrow \infty}\left|V\left(\phi_{n}\right)\right| \\
& =\sup _{\left\{\phi_{n}\right\} \in Q_{d}(R)} \limsup _{n \rightarrow \infty}\left|\int_{R} \mu \phi_{n}\right|,
\end{aligned}
$$

where $\mu$ represents $\widehat{V}$. In particular, if $\widehat{V} \in Z_{0}(R)$, it holds

$$
b(\widehat{V})=\sup _{\left\{\phi_{n}\right\} \in Q_{d}(R)} \limsup _{n \rightarrow \infty}\left|\int_{R} \mu \phi_{n}\right|=0 .
$$

The infinitesimal asymptotic Teichmüller distance between two points $\widehat{V}_{1}$ and $\widehat{V}_{2}$ in $A Z(R)$ is defined as

$$
\begin{aligned}
\bar{d}\left(\widehat{V}_{1}, \widehat{V}_{2}\right) & =b\left(\widehat{V}_{1}-\widehat{V}_{2}\right) \\
& =\sup _{\left\{\phi_{n}\right\} \in Q_{d}(R)} \limsup _{n \rightarrow \infty}\left|\int_{R}(\mu-\nu) \phi_{n}\right|,
\end{aligned}
$$

where $\mu$ and $\nu$ represent $V_{1}$ and $V_{2}$, respectively.

For any $V \in Z(\mathrm{R}), \mu \in L^{\infty}(R)$ is an infinitesimal extremal representative of $V$ if and only if it has a so-called Hamilton sequence, namely, a sequence $\left\{\phi_{n}\right\} \in Q_{1}(R)$, such that

$$
\lim _{n \rightarrow \infty}\left|\int_{R} \mu \phi_{n}\right|=\|\mu\|_{\infty} .
$$

Similarly, for any $\widehat{V} \in A Z(R), \mu \in L^{\infty}(R)$ is an infinitesimal asymptotically extremal representative of $\widehat{V}[1,6]$ if and only 
if there exists an asymptotic Hamilton sequence of $\mu$, namely, a degenerating sequence $\left\{\phi_{n}\right\} \subset Q_{d}(R)$, such that

$$
\lim _{n \rightarrow \infty}\left|\int_{R} \mu \phi_{n}\right|=b^{*}(\mu) .
$$

Let $\mathbb{D}=\{z:|z|<1\}$ be the unit disk in the extended complex plane $\widehat{\mathbb{C}}$ and let $\partial \mathbb{D}$ be the unit circle. In the following part, we consider some results about the infinitesimal local boundary dilatation of $V$ in the tangent space $Z(\mathbb{D})$ to the universal Teichmüller space.

Set $p \in \partial \mathbb{D}$ and $U_{r}(p)=\mathbb{D} \cap\{z:|z-p|<r\}$. For any $V \in Z(\mathbb{D})$, the infinitesimal local boundary dilatation of $V$ at $p$ is defined as

$$
b_{p}(V)=b_{p}\left([\mu]_{z}\right)=\inf \left\{b_{p}^{*}(\mu): \mu \text { represents } V\right\},
$$

where

$$
b_{p}^{*}(\mu)=\inf _{r}\left\{\left\|\left.\mu(z)\right|_{U_{r}(p)}\right\|_{\infty}: z \in U_{r}(p)\right\} .
$$

For any $\widehat{V} \in A Z(\mathbb{D})$, we define

$$
b_{p}(\widehat{V})=b_{p}\left([[\mu]]_{z}\right)=\inf \left\{b_{p}^{*}(\mu): \mu \text { represents } \widehat{V}\right\} \text {, }
$$

and then $b_{p}(V)=b_{p}(\widehat{V})$. It is proved by Lakic [16] that

$$
b(\widehat{V})=\max _{p \in \partial \mathbb{D}} b_{p}(\widehat{V}) .
$$

A point $p \in \partial \mathbb{D}$ with $b_{p}(\widehat{V})=b(\widehat{V})$ is said to be an infinitesimal substantial boundary point for $\widehat{V} \cdot \widehat{V}$ (or $[[\mu]]_{z}$ ) is called an infinitesimal substantial point in $A Z(\mathbb{D})$, if every $p \in \partial \mathbb{D}$ is an infinitesimal substantial boundary point for $\widehat{V}$ (or $[[\mu]]_{z}$ ); otherwise, $\widehat{V}$ (or $[[\mu]]_{z}$ ) is called an infinitesimal nonsubstantial point.

The following lemma can be obtained in [17] by Fehlmann and Sakan.

Lemma 1. For any $V \in Z(\mathbb{D})$, let $\mu \in L^{\infty}(\mathbb{D})$ be an infinitesimal extremal representative of $V$. Suppose there is a point $p \in \partial \mathbb{D}$ which is not a substantial boundary point of $V$. Then, there is an open interval $I \subset \partial \mathbb{D}, p \in I$, and a domain

$$
S_{I}=\left\{z \in \mathbb{D} \mid z \neq 0, \frac{z}{|z|} \in I\right\},
$$

such that, for any degenerating Hamilton sequence $\left\{\psi_{n}\right\}$ of $\mu$, one has

$$
\lim _{n \rightarrow \infty} \int_{S_{I}}\left|\psi_{n}\right| d x d y=0
$$

\section{Constructing a Special Sequence Degenerating towards a Boundary Point}

In this section, we will construct a special sequence degenerating towards a boundary point $p \in \partial \mathbb{D}$. The method used here is similar to that in [18] while the sequence degenerates towards the whole boundary in this paper.
Let $p \in \partial \mathbb{D}$ and $U_{r}=\{z \in \mathbb{D}:|z-p|<r\}$. A degenerating sequence $\left\{\phi_{n}\right\} \subset Q_{d}(\mathbb{D})$ is said to degenerate towards $p$ if, for any neighbourhood $U_{r}$ of $p$ with $r>0$,

$$
\lim _{n \rightarrow \infty} \int_{U_{r}(p)}\left|\phi_{n}(z)\right| d x d y=1 .
$$

Then, for any $\epsilon>0$ and $r>0$, there exists a positive integer $N$, such that

$$
1-\epsilon<\int_{U_{r}}\left|\phi_{n}(z)\right| d x d y<1
$$

holds for every $n>N$. Since $\left\|\phi_{n}\right\|=1, \forall n \in \mathbb{N}$, there exists a positive number $r_{1}<1$ satisfying

$$
\int_{\mathbb{D} \backslash D_{r_{1}}}\left|\phi_{1}\right|>1-\frac{1}{2} .
$$

By the definition of degenerating sequence and (26), there exists $\phi_{k_{2}}$ such that

$$
\begin{aligned}
\left|\phi_{k_{2}}\right| & <\frac{1}{2^{2}}, \text { for }|z| \leq 1-r_{1}, \\
\int_{\mathbb{D} \backslash U_{r_{1}}}\left|\phi_{k_{2}}\right| & <\frac{1}{4^{2}} .
\end{aligned}
$$

Choose a positive number $r_{2}<r_{1}$ such that

$$
\begin{gathered}
\int_{\mathbb{D} \mid U_{r_{2}}}\left|\phi_{k_{2}}\right|>1-\frac{1}{4^{2}}, \\
\int_{U_{r_{2}}}\left|\phi_{k_{1}}\right|<\frac{1}{2^{2}},
\end{gathered}
$$

where $k_{1}=1$. It follows from (29) and (30) that

$$
\int_{U_{r_{1}} \backslash U_{r_{2}}}\left|\phi_{k_{2}}\right|>1-\frac{1}{2^{2}}
$$

By induction, we obtain a subsequence $\left\{\phi_{k_{n}}\right\}$ of $\left\{\phi_{k}\right\}$ and a positive number sequence $\left\{r_{n}\right\}$ with $r_{n}<r_{n-1}$ and $\lim _{n \rightarrow \infty} r_{n}=0$ such that

$$
\begin{aligned}
\left|\phi_{k_{n}}\right| & <\frac{1}{2^{n}}, \quad \text { in }\left(|z| \leq 1-r_{n}\right), \\
\int_{U_{r_{n-1}} \mid U_{r_{n}}}\left|\phi_{k_{n}}\right| & >1-\frac{1}{2^{n}}, \\
\int_{U_{r_{n}}}\left|\phi_{k_{j}}\right| & <\frac{1}{2^{n}}, \quad j=1,2, \ldots, n-1,
\end{aligned}
$$

where $n=2,3, \ldots$

Without loss of generality, from now on, we write $\phi_{n}$ instead of $\phi_{k_{n}}$ for simplicity. Let

$$
\phi=\sum_{n=1}^{\infty} \phi_{n} .
$$


From (33), it is easy to see that this series is uniformly convergent in every compact subset of $\mathbb{D}$. So $\phi$ is a holomorphic quadratic differential on $\mathbb{D}$.

Noting that $\left\|\phi_{n}\right\|=1$ and by (34), we get

$$
\begin{aligned}
\int_{U_{r_{n}}}\left|\phi_{n}\right| & \leq \frac{1}{2^{n}}, \\
\int_{\mathbb{D} \mid U_{r_{n-1}}}\left|\phi_{n}\right| & \leq \frac{1}{2^{n}} ;
\end{aligned}
$$

that is,

$$
\begin{gathered}
\int_{U_{r_{n}}}\left|\phi_{n}\right|=O\left(2^{-n}\right), \quad n \longrightarrow \infty, \\
\int_{\mathbb{D} \backslash U_{r_{n-1}}}\left|\phi_{n}\right|=O\left(2^{-n}\right), \quad n \longrightarrow \infty .
\end{gathered}
$$

Moreover, by the second formula of (37), we have

$$
\int_{\mathbb{D} \backslash U_{r_{n}}}\left|\phi_{j}\right| \leq \frac{1}{2^{j}}, \quad j \geq n+1
$$
that

By simple calculation, it follows from (35), (37), and (39)

$$
\begin{aligned}
\int_{U_{r_{n-1}} \backslash U_{r_{n}}}\left|\phi-\phi_{n}\right| \leq & \sum_{j=1}^{n-1} \int_{U_{r_{n-1}} \mid U_{r_{n}}}\left|\phi_{j}\right| \\
& +\sum_{j=n+1}^{\infty} \int_{U_{r_{n-1}} \mid U_{r_{n}}}\left|\phi_{j}\right| \\
& <\sum_{j=1}^{n-1} \int_{U_{r_{n-1}}}\left|\phi_{j}\right|+\sum_{j=n+1}^{\infty} \int_{\mathbb{D} \mid U_{r_{n}}}\left|\phi_{j}\right| \\
& <\sum_{j=1}^{n-1} \frac{1}{2^{n-1}}+\sum_{j=n+1}^{\infty} \frac{1}{2^{j}}<\frac{n}{2^{n-1}} .
\end{aligned}
$$

Then,

$$
\int_{U_{r_{n-1}} \mid U_{r_{n}}}\left|\phi-\phi_{n}\right|=O\left(n 2^{-(n-1)}\right), \quad n \longrightarrow \infty .
$$

Furthermore, from (34) and (41), we have

$$
\int_{U_{r_{n-1}} \backslash U_{r_{n}}}|\phi|=1+O\left(n 2^{-(n-1)}\right), \quad n \longrightarrow \infty .
$$

\section{Nonuniqueness of Geodesics Joining Every Infinitesimal Nonsubstantial Point with the Basepoint in $A Z(\mathbb{D})$}

For every $\widehat{V} \in A Z(R)$, it is known in [1] that there exists a representative $\mu$ such that $\|\mu\|_{\infty}=b(\widehat{V})$. Then

$$
\|\mu\|_{\infty}=b(\widehat{V}) \leq\|V\| \leq\|\mu\|_{\infty}
$$

means $\mu$ is an infinitesimal extremal representative of $\widehat{V}$, and

$$
\|\mu\|_{\infty}=b(\widehat{V}) \leq b^{*}(\mu) \leq\|\mu\|_{\infty}
$$

implies $\mu$ is an infinitesimal asymptotic extremal representative of $\widehat{V}$. Set $b(\widehat{V})=k$ and

$$
\gamma(t):[0, k] \longrightarrow A Z(R) ; \quad t \longmapsto\left[\left[\frac{t \mu}{k}\right]\right]_{z} .
$$

It is clear that $\gamma(0)=[[0]]_{z}$ and $\gamma(k)=[[\mu]]_{z}$; moreover, $\gamma([0, k])$ is a geodesic segment joining 0 and $\widehat{V}$ in $A Z(R)$.

Let $p \in \partial \mathbb{D}$ and $U_{r}(p)=\{z \in \mathbb{D}:|z-p|<$ $r\}$. In this section, we will discuss the nonuniqueness of geodesic segments between 0 and $\widehat{V}$ in $A Z(\mathbb{D})$ when $\widehat{V}$ is an infinitesimal nonsubstantial point; that is, there exists a point $p \in \partial \mathbb{D}$ with $b_{p}(\widehat{V})<b(\widehat{V})$.

We need the following important lemma.

Lemma 2. Let $\widehat{V} \in A Z(\mathbb{D})$ and $p \in \partial \mathbb{D}$. Then, for any given $\epsilon>0$, there exists an infinitesimal asymptotic extremal representative $\mu$ of $\widehat{V}$ such that

$$
b_{p}^{*}(\mu)<b_{p}(\widehat{V})+\epsilon .
$$

Proof. Suppose $p$ is an infinitesimal substantial boundary point for $\widehat{V}$; that is, $b_{p}(\widehat{V})=b(\widehat{V})$. It is known in [6] that there exists an infinitesimal asymptotic extremal representative $\mu \epsilon$ $L^{\infty}(\mathbb{D})$ such that $b(\widehat{V})=b^{*}(\mu)$. We conclude that (46) holds since

$$
b_{p}^{*}(\mu) \leq b^{*}(\mu)=b(\widehat{V})=b_{p}(\widehat{V}) \text {. }
$$

Otherwise, suppose $b_{p}(\widehat{V})<b(\widehat{V})$. For any $\epsilon>0$, without loss of generality, we assume that $\epsilon<b(\widehat{V})-b_{p}(\widehat{V})$. By the definition of the infinitesimal local boundary dilatation, there exists a Beltrami differential $\mu$ representing $\widehat{V}$ such that

$$
b_{p}^{*}(\mu)<b_{p}(\widehat{V})+\frac{\epsilon}{2}<b(\widehat{V}) .
$$

Moreover, by the definition of $b_{p}^{*}(\mu)$, there exists $r_{0}>0$ such that

$$
\left\|\left.\mu\right|_{U_{r_{0}}(p)}\right\|_{\infty}<b_{p}^{*}(\mu)+\frac{\epsilon}{2} .
$$

Let $v$ be an infinitesimal extremal and asymptotic extremal representative of $\widehat{V}$; that is, $\|\nu\|_{\infty}=b(\widehat{V})$, and let $\left\{\phi_{n}\right\} \subset Q_{d}(R)$ be an asymptotic Hamilton sequence of $\nu$. By Lemma $1\left(r<r_{0}\right.$ sufficiently small), we have

$$
\lim _{n \rightarrow \infty} \int_{U_{r}(p)}\left|\phi_{n}\right| d x d y=0 .
$$

So

$$
\lim _{n \rightarrow \infty}\left|\int_{U_{r}(p)} \nu \phi_{n} d x d y\right| \leq\|\nu\|_{\infty} \lim _{n \rightarrow \infty} \int_{U_{r}(p)}\left|\phi_{n}\right| d x d y
$$


which means

$$
\lim _{n \rightarrow \infty} \int_{U_{r}(p)} \nu \phi_{n} d x d y=0
$$

There exists a boundary point $p^{\prime} \in \partial \mathbb{D}$ outside $U_{r}(p)$ satisfying

$$
b_{p^{\prime}}(\widehat{V})=b(\widehat{V})
$$

so we have $b_{p^{\prime}}^{*}(v)=b^{*}(v)$. Let $\chi$ be the characteristic function and $\widetilde{v}=\nu \chi_{\mathbb{D} \backslash U_{r}(p)}$. Then

$$
b^{*}(\widetilde{\nu})=b^{*}(\nu)=b(\widehat{V}) .
$$

Since

$$
\int_{\mathbb{D}} \nu \phi_{n} d x d y=\int_{\mathbb{D}} \tilde{v} \phi_{n} d x d y+\int_{U_{r}(p)} \nu \phi_{n} d x d y,
$$

we get

$$
\lim _{n \rightarrow \infty} \int_{\mathbb{D}} \tilde{v} \phi_{n} d x d y=\lim _{n \rightarrow \infty} \int_{\mathbb{D}} \nu \phi_{n} d x d y,
$$

due to (52). It follows from (18) and (54) that

$$
\begin{aligned}
\lim _{n \rightarrow \infty}\left|\int_{\mathbb{D}} \tilde{\nu} \phi_{n} d x d y\right| & =\lim _{n \rightarrow \infty}\left|\int_{\mathbb{D}} \nu \phi_{n} d x d y\right|=b(\widehat{V}) \\
& =b^{*}(\widetilde{\nu}) .
\end{aligned}
$$

So $\widetilde{v}$ is an infinitesimal asymptotically extremal Beltrami differential in its equivalence class $[[\widetilde{\nu}]]_{z}$ and $b\left([[\widetilde{\nu}]]_{z}\right)=b(\widehat{V})$.

Define

$$
\tilde{\mu}(z)= \begin{cases}\widetilde{v}(z), & z \in \mathbb{D} \backslash U_{r}(p) \\ \mu(z), & z \in U_{r}(p) .\end{cases}
$$

It is not hard to verify that $\tilde{\mu}(z)$ is an infinitesimal asymptotically extremal representative of $\widehat{V}$, and by (48) and (49), $b_{p}^{*}(\widetilde{\mu})<b_{p}(\widehat{V})+\epsilon$. This completes the proof of Lemma 2.

Theorem 3. For every $\widehat{V} \in A Z(\mathbb{D})$, if $\widehat{V}$ is a nonsubstantial point, that is, $b_{p}(\widehat{V})<b(\widehat{V})$ for some point $p \in \partial \mathbb{D}$, then there exist infinitely many geodesic segments connecting $\widehat{V}$ and the basepoint in $A Z(\mathbb{D})$.

Proof. Let $\epsilon=b(\widehat{V})-b_{p}(\widehat{V})$. By Lemma 2, there exists an infinitesimal asymptotic extremal representative $\mu$ of $\widehat{V}$ such that

$$
b_{p}^{*}(\mu)<b_{p}(\widehat{V})+\epsilon .
$$

So there exists a positive number $r_{0}$ and a neighbourhood $U_{r_{0}}=\left\{z \in \mathbb{D}:|z-p|<r_{0}\right\}$ of $p$ such that

$$
\left\|\left.\mu\right|_{U_{r_{0}}}\right\|_{\infty}<b(\widehat{V})
$$

Set $b(\widehat{V})=k,\left\|\left.\mu\right|_{U_{r_{0}}}\right\|_{\infty}=k^{\prime}$ and $\delta=1-k^{\prime} / k$. Since $\mu$ is infinitesimally asymptotically extremal, $b^{*}(\mu)=b(V)=$ $k$. From (18), there exists an asymptotic Hamilton sequence $\left\{\psi_{n}\right\} \subset Q_{d}(R)$ such that

$$
\lim _{n \rightarrow \infty}\left|\int_{R} \mu \psi_{n}\right|=b^{*}(\mu)
$$

Furthermore, by Lemma 1, we have

$$
\lim _{n \rightarrow \infty} \int_{U_{r_{0}}}\left|\psi_{n}\right|=0
$$

for sufficiently small $r_{0}$.

Let $\chi_{r_{0}}$ be the characteristic function of $U_{r_{0}}$ and let $\phi_{n}$ be the special sequence degenerating towards $p$ constructed as above. For every $0 \leq \rho \leq \delta$ and $t \in[0, k]$, let

$$
\gamma_{\rho}(t)=\left[\left[\frac{t \mu}{k}+\frac{(\rho / 2) t(k-t) \chi_{r_{0}}|\phi|}{\phi}\right]\right]_{z},
$$

where $\phi=\sum_{n=1}^{\infty} \phi_{n}$ is a holomorphic quadratic differential on $\mathbb{D}$. Clearly, $\gamma_{\rho}(0)=[[0]]_{z}$ and $\gamma_{\rho}(k)=[[\mu]]_{z}$.

Now we show that $\gamma_{\rho}([0, k])$ is a geodesic segment in $A Z(\mathbb{D})$. Let $0 \leq t_{1} \leq t_{2} \leq k$. We discuss the infinitesimally asymptotic equivalence class

$$
\left[\left[\left(t_{2}-t_{1}\right)\left(\frac{\mu}{k}+\frac{(\rho / 2)\left(k-t_{1}-t_{2}\right) \chi_{r_{0}}|\phi|}{\phi}\right)\right]\right]_{z} .
$$

By Lemma 1, it is easy to calculate that

$$
\begin{aligned}
& \lim _{n \rightarrow \infty} \mid \int_{\mathbb{D}}\left(t_{2}-t_{1}\right)\left(\frac{\mu}{k}+\frac{(\rho / 2)\left(k-t_{1}-t_{2}\right) \chi_{r}|\phi|}{\phi}\right) \\
& \cdot \psi_{n} \mid=t_{2}-t_{1} .
\end{aligned}
$$

Since

$$
\begin{aligned}
& b^{*}\left(\left(t_{2}-t_{1}\right)\left(\frac{\mu}{k}+\frac{(\rho / 2)\left(k-t_{1}-t_{2}\right) \chi_{r_{0}}|\phi|}{\phi}\right)\right) \\
& \quad=t_{2}-t_{1},
\end{aligned}
$$

we obtain that

$$
\left(t_{2}-t_{1}\right)\left(\frac{\mu}{k}+\frac{(\rho / 2)\left(k-t_{1}-t_{2}\right) \chi_{r_{0}}|\phi|}{\phi}\right)
$$

is infinitesimally asymptotically extremal in its equivalence class. From (16), we have

$$
\begin{aligned}
& \bar{d}\left(\gamma_{\rho}\left(t_{1}\right), \gamma_{\rho}\left(t_{2}\right)\right)=b\left(\left[\left[\left(t_{2}-t_{1}\right)\right.\right.\right. \\
& \left.\left.\left.\cdot\left(\frac{\mu}{k}+\frac{(\rho / 2)\left(k-t_{1}-t_{2}\right) \chi_{r_{0}}|\phi|}{\phi}\right)\right]\right]_{z}\right)=t_{2} \\
& -t_{1}
\end{aligned}
$$


which implies that $\gamma_{\rho}([0, k])$ is a geodesic segment joining $[[0]]_{z}$ and $[[\mu]]_{z}$ in $A Z(\mathbb{D})$.

Now we prove that, for $0 \leq \rho_{1}<\rho_{2} \leq \delta, \gamma_{\rho_{1}}([0, k])$ and $\gamma_{\rho_{2}}([0, k])$ are two different geodesics joining $[[0]]_{z}$ and $[[\mu]]_{z}$ in $A Z(\mathbb{D})$. Otherwise, suppose $\gamma_{\rho_{1}}(t)=\gamma_{\rho_{2}}(t), t \in(0, k)$ when $\rho_{1}<\rho_{2}$. Then

$$
\left[\left[\frac{\left(\left(\rho_{2}-\rho_{1}\right) / 2\right) t(k-t) \chi_{r_{0}}|\phi|}{\phi}\right]\right]_{z} \in Z_{0}(\mathbb{D}) .
$$

From (15), for the special sequence $\phi_{n}$ degenerating towards $p$ constructed as above, it yields

$$
\lim _{n \rightarrow \infty}\left|\int_{\mathbb{D}} \chi_{r_{0}} \frac{|\phi|}{\phi} \phi_{n}\right|=0
$$

On the other hand, there exists a positive number sequence $\left\{r_{n}\right\}$ corresponding to $\left\{\phi_{n}\right\}$ with $r_{n}<r_{n-1}<\cdots<r_{0}$ and $\lim _{n \rightarrow \infty} r_{n}=0$ such that

$$
\begin{aligned}
\int_{\mathbb{D}} \chi_{r_{0}} \frac{|\phi|}{\phi} \phi_{n}= & \int_{U_{r_{0}} \backslash U_{r_{n-1}}} \frac{|\phi|}{\phi} \phi_{n}+\int_{U_{r_{n-1} \backslash U_{r_{n}}}} \frac{|\phi|}{\phi} \phi_{n} \\
& +\int_{U_{r_{n}}} \frac{|\phi|}{\phi} \phi_{n} .
\end{aligned}
$$

It follows from (38) that

$$
\begin{aligned}
& \lim _{n \rightarrow \infty}\left|\int_{U_{r_{n}}} \frac{|\phi|}{\phi} \phi_{n}\right| \leq \lim _{n \rightarrow \infty} \int_{U_{r_{n}}}\left|\phi_{n}\right|=0, \\
& \lim _{n \rightarrow \infty}\left|\int_{U_{r_{0}} \backslash U_{r_{n-1}}} \frac{|\phi|}{\phi} \phi_{n}\right| \leq \lim _{n \rightarrow \infty} \int_{U_{r_{0}} \mid U_{r_{n-1}}}\left|\phi_{n}\right|=0 .
\end{aligned}
$$

So

$$
\begin{array}{r}
\lim _{n \rightarrow \infty} \int_{U_{r_{n}}} \frac{|\phi|}{\phi} \phi_{n}=0, \\
\lim _{n \rightarrow \infty} \int_{U_{r_{0}} \backslash U_{r_{n-1}}} \frac{|\phi|}{\phi} \phi_{n}=0 .
\end{array}
$$

Furthermore,

$$
\begin{aligned}
\int_{U_{r_{n-1}} \backslash U_{r_{n}}} \frac{|\phi|}{\phi} \phi_{n}= & \int_{U_{r_{n-1}} \backslash U_{r_{n}}} \frac{|\phi|}{\phi} \phi \\
& +\int_{U_{r_{n-1}} \backslash U_{r_{n}}} \frac{|\phi|}{\phi}\left(\phi_{n}-\phi\right) .
\end{aligned}
$$

It follows from (41) and (42) that

$$
\begin{array}{r}
\lim _{n \rightarrow \infty} \int_{U_{r_{n-1}} \backslash U_{r_{n}}} \frac{|\phi|}{\phi}\left(\phi_{n}-\phi\right)=0, \\
\lim _{n \rightarrow \infty} \int_{U_{r_{n-1}} \backslash U_{r_{n}}}|\phi|=1 .
\end{array}
$$

Therefore,

$$
\lim _{n \rightarrow \infty} \int_{U_{r_{n-1}} \backslash U_{r_{n}}} \frac{|\phi|}{\phi} \phi_{n}=1
$$

By (71)-(76), we conclude that

$$
\lim _{n \rightarrow \infty}\left|\int_{\mathbb{D}} \chi_{r_{0}} \frac{|\phi|}{\phi} \phi_{n}\right|=1 .
$$

This is a contradiction with (70), which implies $\gamma_{\rho_{1}}(t) \neq \gamma_{\rho_{2}}(t)$ for $t \in(0, k)$ if $\rho_{1} \neq \rho_{2}$.

Thus, we have constructed infinitely many geodesics $\gamma_{\rho}(t)$ $(0 \leq \rho \leq \delta)$ joining $[[0]]$ and $[[\mu]]$ in $A Z(\mathbb{D})$.

The situation on the geodesics joining an infinitesimal substantial point with the basepoint is not clear. We conjecture that there exist infinitely many geodesics between an infinitesimal substantial point and the basepoint in $A Z(\mathbb{D})$.

\section{Conflict of Interests}

The authors declare that there is no conflict of interests regarding the publication of this paper.

\section{Acknowledgment}

The research is partially supported by the National Natural Science Foundation of China (Grants nos. 11371045, 11301248, and 11271175).

\section{References}

[1] C. J. Earle, F. P. Gardiner, and N. Lakic, "Asymptotic Teichmüller space. Part II: The metric structure," Contemporary Mathematics, vol. 355, pp. 187-219, 2004.

[2] K. Astala and M. Zinsmeister, "Teichmüller spaces and BMOA," Mathematische Annalen, vol. 289, no. 4, pp. 613-625, 1991.

[3] G. Cui, "Integrably asymptotic affine homeomorphisms of the circle and Teichmüller spaces," Science in China Series A: Mathematics, vol. 43, no. 3, pp. 267-279, 2000.

[4] A. Fletcher, "On asymptotic Teichmüller space," Transactions of the American Mathematical Society, vol. 362, no. 5, pp. 25072523, 2010.

[5] H. Miyachi, "On invariant distances on asymptotic Teichmüller spaces," Proceedings of the American Mathematical Society, vol. 134, no. 7, pp. 1917-1925, 2006.

[6] F. P. Gardiner and N. Lakic, Quasiconformal Teichmüller Theory, American Mathematical Society, Providence, RI, USA, 2000.

[7] O. Lehto, Univalent Functions and Teichmüller Spaces, Springer, New York, NY, USA, 1986.

[8] S. Nag, The Complex Analytic Theory of Teichmüller Spaces, Wiley-Interscience, 1988.

[9] C. J. Earle, I. Kra, and S. L. Krushkal, "Holomorphic motions and teichmüller spaces," Transactions of the American Mathematical Society, vol. 343, no. 2, pp. 927-948, 1994.

[10] J. Fan, "On geodesics in asymptotic Teichmüller spaces," Mathematische Zeitschrift, vol. 267, no. 3-4, pp. 767-779, 2011. 
[11] Z. Li, "Nonuniqueness of geodesics in infinite dimensional Teichmüller spaces," Complex Variables, Theory and Application, vol. 16, no. 4, pp. 261-272, 1991.

[12] Z. Li, "Non-uniqueness of geodesics in infinite dimensional Teichmüller spaces (II)," Annales Academioe Scientiarum Fennica Mathematica, vol. 18, pp. 335-367, 1993.

[13] Y. L. Shen, "Some remarks on the geodesics in infinitedimensional Teichmüller spaces," Acta Mathematica Sinica, vol. 13, no. 4, pp. 497-502, 1997.

[14] Y.-L. Shen, "On Teichmüller geometry," Complex Variables, Theory and Application, vol. 44, no. 1, pp. 73-83, 2001.

[15] H. Tanigawa, "Holomorphic families of geodesic discs in infinite-dimensional Teichmüller spaces," Nagoya Mathematical Journal, vol. 127, pp. 117-128, 1992.

[16] N. Lakic, "Substantial boundary points for plane domains and Gardiner's conjecture," Annales Academioe Scientiarum Fennicae Mathematica, vol. 25, pp. 285-306, 2000.

[17] R. Fehlmann and K.-I. Sakan, "On the set of substantial boundary points for extremal quasiconformal mappings," Complex Variables, Theory and Application, vol. 6, no. 2-4, pp. 323-335, 1986.

[18] Z. Li, "A note on geodesics in infinite-dimensional Teichmüller spaces," Annales Academiae Scientiarum Fennicae. Series A I. Mathematica, vol. 20, no. 2, pp. 301-313, 1995. 


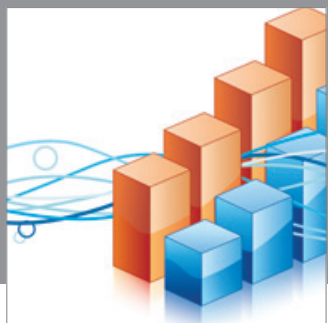

Advances in

Operations Research

mansans

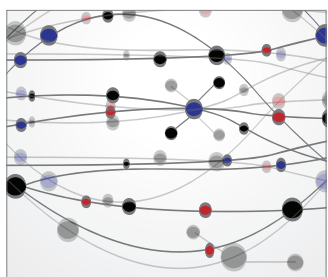

The Scientific World Journal
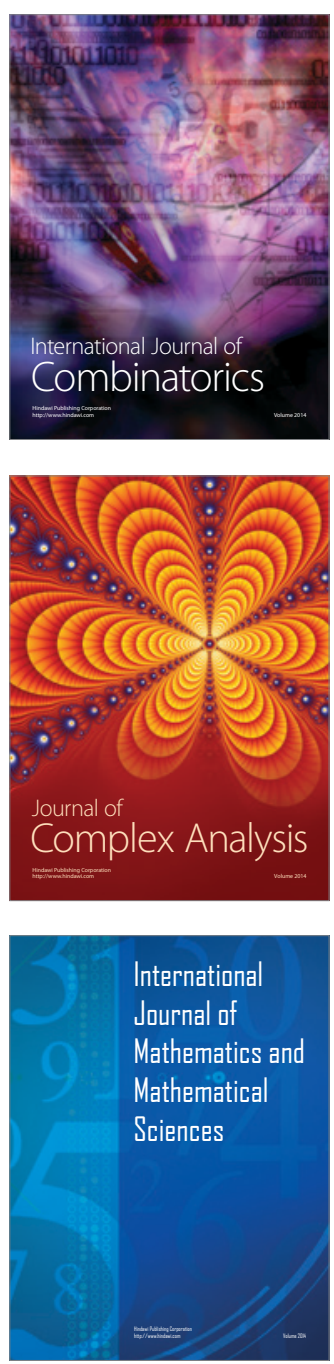
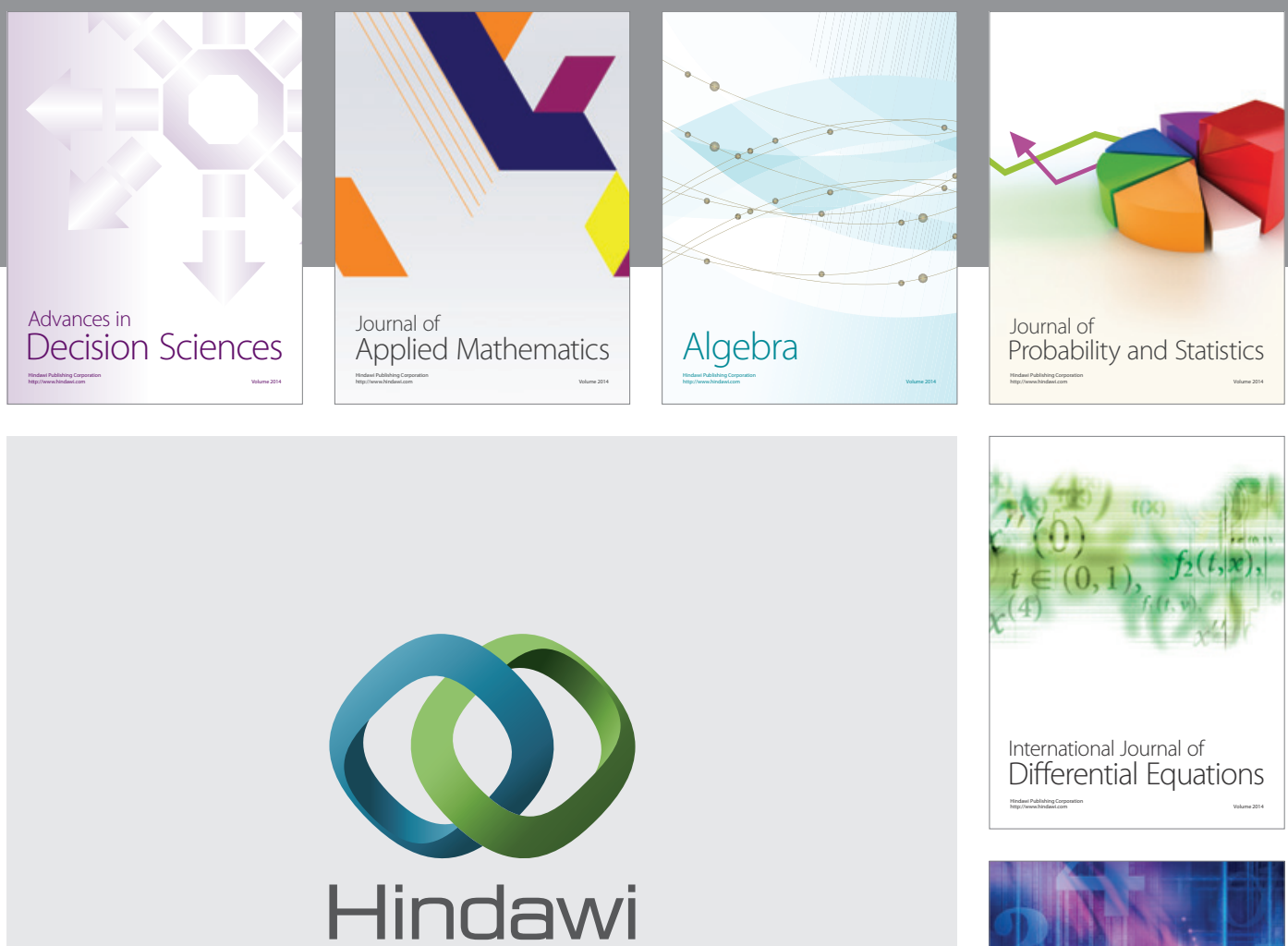

Submit your manuscripts at http://www.hindawi.com
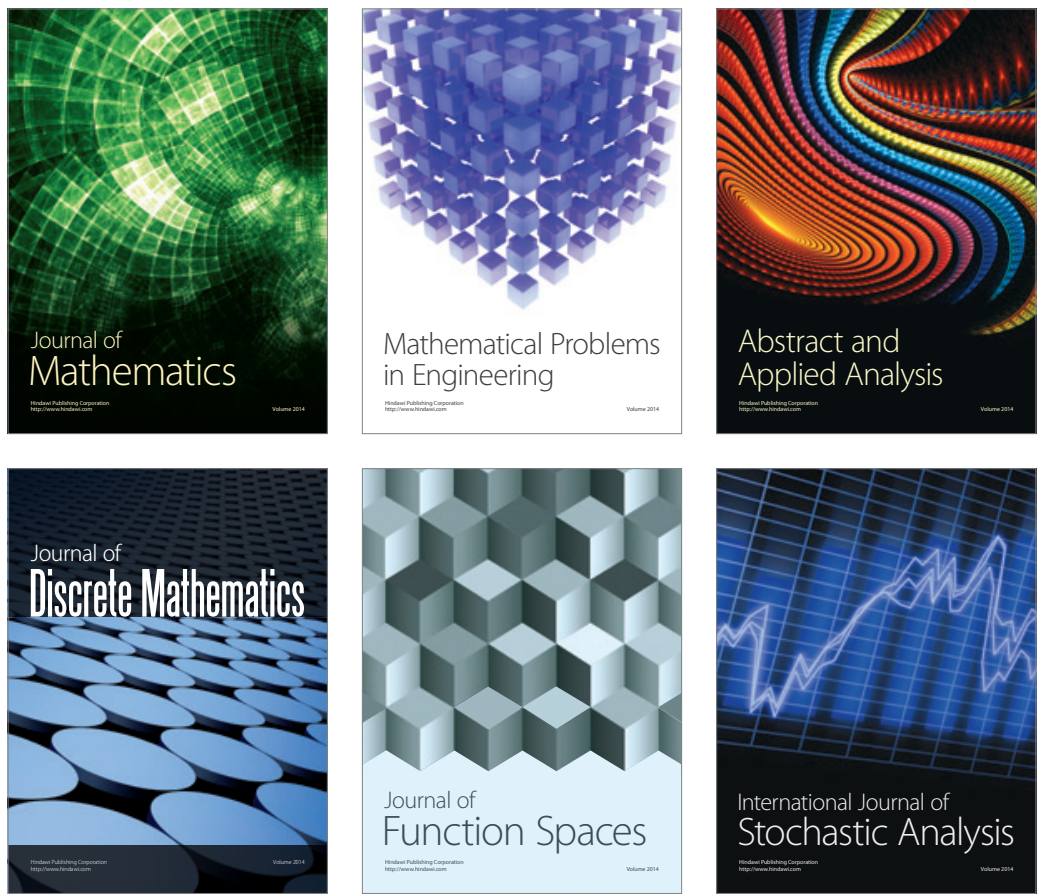

Journal of

Function Spaces

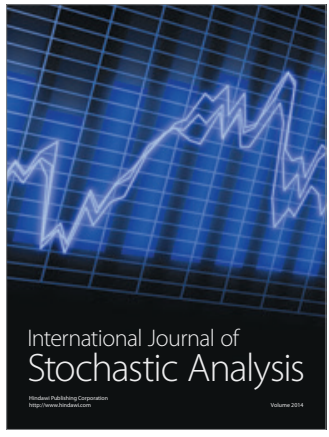

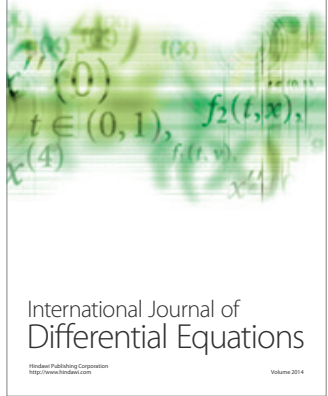
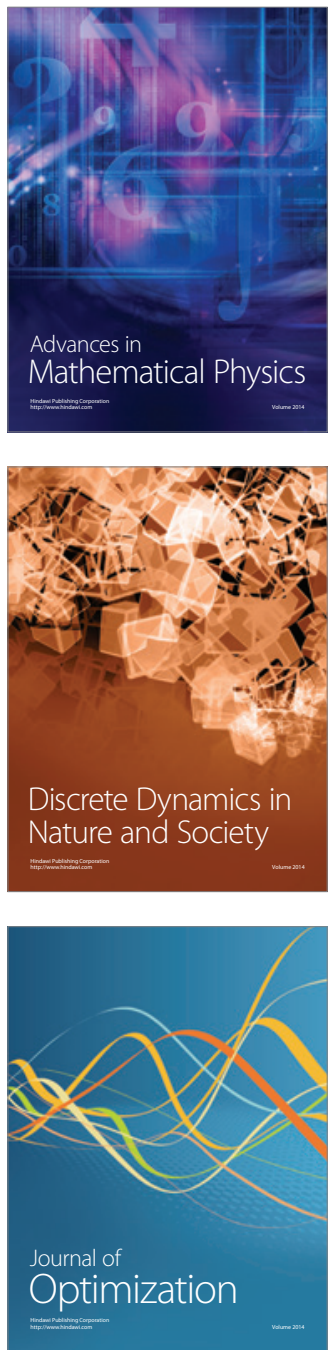